\title{
Propuesta de actividades de enseñanza - aprendizaje e instrumentos de evaluación de competencias en el Máster Universitario en Ingeniería de Análisis de Datos, Mejora de Procesos y Toma de Decisiones
}

\author{
Andrea Conchado Peiróa, Ana Isabel Sánchez Galdóna , Rubén Ruiz García ${ }^{a}$, María \\ del Carmen Bas Cerdáa, Elena Vázquez Barrachina ${ }^{a}$, Rosa María Alcover Arándiga ${ }^{a}$, \\ Andrés Carrión García ${ }^{a}$ y José Miguel Carot Sierra ${ }^{\mathrm{a}}$ \\ ${ }^{a}$ Departamento de Estadística e Investigación Operativa Aplicadas y Calidad
}

\begin{abstract}
The present paper aims to set a systematic strategy of competences assessment comprising the Master's Degree in Data Analysis, Process Improvement and Decision Support Engineering, offered by the department of Applied Statistics and Operational Research, and Quality of the UPV. The purpose of this innovation is to facilitate the competence accreditation of graduates at individual level, as well as study programme level. To this end, a review of the competencies offered by Master's degree was conducted, matching these specific degree competences with UPV's “transversal” competences. This study has allowed the assignment of learning outcomes or competences to specific subjects of the degree. In this sense, two new activities for the teaching - learning of transversal competence "Analysis and troubleshooting”, as well as for the specific competence "Development, Construction, Validation and Interpretation of Models" were set out. In addition, a rubric for assessing these two competences was built. Finally, we have identified significant differences between the marks obtained by students in the two activities.
\end{abstract}

Keywords: "transversal" competences, data analysis, assessment tools, methodologies

\footnotetext{
Resumen

El presente trabajo pretende definir una estrategia sistemática de evaluación de competencias para el Máster Universitario en Ingeniería de Análisis de Datos, Mejora de Procesos y Toma de Decisiones, ofertado por el Departamento de Estadística e Investigación Operativa Aplicadas y Calidad
} 
Propuesta de actividades de enseñanza - aprendizaje e instrumentos de evaluación de competencias en el Máster Universitario en Ingeniería de Análisis de Datos, Mejora de Procesos y Toma de Decisiones

de la UPV. El objetivo de esta innovación es facilitar la acreditación de competencias a los egresados de la titulación de forma individual, así como a nivel de promoción anual. Para ello se ha realizado una revisión de las competencias ofertadas por la titulación y se han establecido correspondencias con las competencias transversales de la UPV. Este estudio ha permitido la asignación de resultados del aprendizaje y/o competencias a determinadas materias del título. Del mismo modo, se ha definido una nueva actividad para la enseñanza - aprendizaje de la competencia transversal Análisis y resolución de problemas y la competencia específica Elaboración, construcción, validación e interpretación de modelos, así como una rúbrica para su evaluación. Finalmente se han identificado diferencias significativas entre las calificaciones obtenidas por los alumnos en dos actos de evaluación.

Palabras clave: competencias transversales, análisis de datos, instrumentos de evaluación, metodologías

\section{Introducción}

El proyecto de competencias transversales coordinado por la Universitat Politècnica de València UPV plantea la necesidad de establecer estrategias de evaluación sistemática de competencias, definiendo dónde y cómo deben ser evaluadas. En la actualidad se están implementando los procedimientos necesarios para llevar a la práctica este objetivo en los estudios de Grado, y está previsto que en los próximos cursos se haga extensible en los estudios de Máster.

En el año 2009 se definieron las competencias asociadas a la titulación del Máster Universitario en Ingeniería de Análisis de Datos, Mejora de Procesos y Toma de Decisiones, que imparte el Departamento de Estadística e Investigación Operativa Aplicadas y Calidad (DEIOAC), durante el proceso de acreditación del título. Concretamente, se especificaron cinco competencias básicas y once competencias generales que podían adquirirse mediante tres módulos obligatorios de materias: análisis de datos, mejora de procesos y toma de decisiones. Sin embargo, hasta la fecha no se ha definido la correspondencia entre estas competencias básicas y generales ofertadas por la titulación y las competencias transversales definidas por la UPV. De acuerdo con el Proyecto Institucional de la Universidad Politécnica de Valencia (2014) sobre competencias transversales, esta modificación se consideraría no sustancial, mientras que la ERT no especificara lo contrario. A simple vista, cabe esperar que se produzcan importantes solapamientos y redundancias entre ambos conjuntos de 
Andrea Conchado Peiró, Ana Isabel Sánchez Galdón, Rubén Ruiz García, María del Carmen Bas Cerdá, Elena Vázquez Barrachina, Rosa María Alcover Arándiga, Andrés Carrión García y José Miguel Carot Sierra

competencias, que permitan adoptar las trece competencias transversales como referencia del título. Por otro lado, se definieron catorce competencias específicas del título, pero recientemente se ha planteado su reformulación, con el fin de facilitar la definición de los resultados del aprendizaje asociados. Esta reordenación interna de las competencias del título permitiría reordenar y plantear nuevas estrategias docentes en base a este conjunto de competencias comunes.

Para que esta reordenación de las competencias del título pueda hacerse efectiva es necesario asignarlas a las distintas materias, de modo que pueda realizarse un análisis, modificación y adaptación, si es necesario, de las guía docentes para incorporar las compentencias transversales, desde el punto de vista metodológico y de contenidos (Villardón, 2015). Una vez realizada esta asignación, el profesorado puede plantear nuevas actividades de enseñanza - aprendizaje e instrumentos de evaluación que se adapten a las guías docentes de cada asignatura (De Miguel, 2006). Así, uno de los objetivos de este trabajo es proponer nuevas actividades piloto con la orientación del Instituto de Ciencias de la Educacuón (ICE) y las lecciones aprendidas de experiencias previas en la UPV, de acuerdo con el principio básico de alineamiento constructivo (Biggs, 2006). Asimismo, los instrumentos de evaluación de dichas competencias serán coherentes con las metodologías previamente empleadas, y orientados en la medida de lo posible a tareas complejas o situaciones - problema (Fernández, 2010).

Las competencias que más se trabajan en las asignaturas en el título, y por tanto constituyen un interesante punto de partida para la definición de actividades de enseñanza aprendizaje e instrumentos de evaluación son Análisis y resolución de problemas (CT3) y Aplicación del pensamiento práctico (CT2). Por otro lado, por el carácter especializado del título, todas las materias trabajan al menos una competencia específica, clasificada dentro de la competencia Instrumental específica (CT13). Este trabajo muestra las actividades e instrumentos desarrollados para el aprendizaje y evaluación de la competencia Análisis y resolución de problemas (CT3) y la competencia específica Elaboración, construcción, validación e interpretación de modelos.

\section{Objetivos}

El objetivo general de esta innovación es plantear un cambio en el enfoque de la evaluación de competencias en la titulación, en sintonía con las competencias transversales propuestas

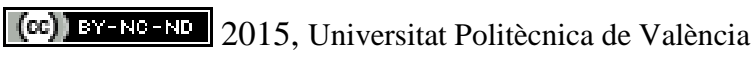

Congreso In-Red (2015) 
Propuesta de actividades de enseñanza - aprendizaje e instrumentos de evaluación de competencias en el Máster Universitario en Ingeniería de Análisis de Datos, Mejora de Procesos y Toma de

Decisiones

por la UPV. Este objetivo forma parte de un proyecto de innovación y mejora educativa en el que colaboran los autores de este trabajo.

Con este fin se definen los siguientes objetivos específicos:

- Establecer una correspondencia entre las competencias básicas y generales ofertadas por la titulación y las competencias transversales definidas por la UPV.

- Asignar una o varias competencias transversales o específicas a determinadas materias, definiendo los resultados del aprendizaje a alcanzar.

- Proponer nuevas actividades piloto de enseñanza - aprendizaje, o modificar las existentes, con el fin de facilitar la adquisición de competencias.

- Seleccionar los instrumentos de evaluación de competencias más adecuados para cada actividad de enseñanza - aprendizaje. 
Andrea Conchado Peiró, Ana Isabel Sánchez Galdón, Rubén Ruiz García, María del Carmen Bas Cerdá, Elena Vázquez Barrachina, Rosa María Alcover Arándiga, Andrés Carrión García y José Miguel Carot Sierra

\section{Desarrollo de la innovación}

Una vez construida la matriz de asociación entre las competencias básicas y generales del título y las trece competencias transversales propuestas por la UPV, que no se muestra en este trabajo por motivos de espacio, se asignó la competencia Análisis y resolución de problemas a las materias Planificación y programación de la producción, Análisis multivariante y Fiabilidad, disponibilidad y mantenibilidad, entre otras asignaturas del título. Esta asigación se decidió en base a la relación de los contenidos con la competencia y la implicación de los profesores que la imparten en el proyecto de innovación y mejora. Estas tres materias contribuyen igualmente a la adquisición de la competencia específica Elaboración, construcción, validación e interpretación de modelos, en colaboración con otras asignaturas del máster.

El principal resultado del aprendizaje definido a nivel de Máster para esta competencia es proponer y construir soluciones a problemas complejos en diversos ámbitos, con una visión global, que se concreta en:

- $\quad$ Identificar problemas con anticipación antes de que su efecto se haga evidente.

- Definir con exactitud el problema que se tiene que resolver, describiendo de manera clara y concisa los hechos más importantes del mismo.

- $\quad$ Descomponer el problema complejo en partes manejables.

- Analizar el problema y sus causas desde un enfoque global.

- Evaluar las posibles soluciones según su viabilidad científico-técnica, económica, temporal, etc.

- Diseñar un plan de acción y seguimiento para la aplicación de la solución escogida.

De acuerdo con la matriz de competencias transversales - metodologías docentes, proporcionada por el ICE, las actividades de enseñanza - aprendizaje recomendadas para el aprendizaje de esta competencias son los problemas en el aula y actividades grupales desarrollados en la lección magistral, así como el aprendizaje basado en problemas o proyectos y las prácticas de laboratorio, planteados como trabajo en equipo o aprendizaje cooperativo.

Las materias implicadas en este trabajo han optado por la metodología de aprendizaje basado en problemas, que de acuerdo con la matriz de metodologías - evaluación proporcionada por el ICE, puede evaluarse mediante observación, examen o exposición oral, redacción de informes escritos, autoevaluación, coevalución entre iguales y portafolio. En este trabajo se muestran los resultados de una actividad de aprendizaje basado en problemas para una de las asignaturas que contribuyen al desarrollo de esta competencia, evaluada

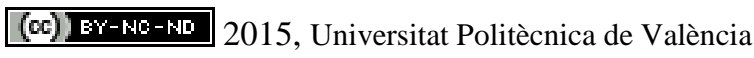

Congreso In-Red (2015) 
Propuesta de actividades de enseñanza - aprendizaje e instrumentos de evaluación de competencias en el Máster Universitario en Ingeniería de Análisis de Datos, Mejora de Procesos y Toma de Decisiones

mediante un informe escrito grupal. Asimismo se muestran resultados estadísticos de las calificaciones obtenidas por los alumnos con una rúbrica diseñada específicamente para esta actividad.

\section{Resultados}

La actividad consiste en el análisis de un problema propuesto por el profesor y la generación de alternativas de solución, aplicando los métodos aprendidos. Los resultados del aprendizaje de esta actividad asociados a la competencia Análisis y Resolución de problemas son:

- $\quad$ Plantear problemas con distintos niveles de dificultad en función de la información disponible

- Seleccionar la infomación necesaria para la resolución del problema

- Generar alternativas de solución mediante la aplicación de los conocimientos y herramientas adquiridos en la asignatura para resolver el problema

- Elaborar un informe escrito sintetizando el proceso de análisis y resolución del problema

- Presentar los resultados del trabajo a un público especializado, como son los compañeros de clase y el profesor

Del mismo modo, esta actividad pretende contribuir al aprendizaje de la competencia específica Elaboración, construcción, validación e interpretación de modelos del título, a través de los resultados del aprendizaje asignados a la asignatura seleccionada para este estudio. La actividad se desarrolla a lo largo de la asignatura y el tiempo estimado para realizarla es 30 horas aproximadamente, distribuidas de la siguiente forma:

- Estudio y trabajo autónomo de los estudiantes para la propuesta de trabajos: 10 horas

- $\quad$ Resolución de las primeras dudas sobre el planteamiento del trabajo con el profesor: 5 horas

- Resolución del problema mediante trabajo en grupo e individual: 10 horas (parte de este trabajo se hace con presencia del profesor en las sesiones prácticas)

- $\quad$ Redacción del informe escrito (memoria): 5 horas

- Presentación oral y respuesta a preguntas del profesor y compañeros: 15 minutos

La duración de la actividad fue 4 semanas, dedicando dos sesiones de 2,5 horas en clase a las mismas. Durante este periodo se realizaron dos actos de evaluación, en cada uno de los cuales los alumnos resolvieron dos problemas independientes. Como recursos, los alumnos tienen a su disposición el material escrito preparado por el profesor, así como ejercicios resueltos,

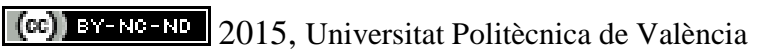

Congreso IN-RED (2015) 
Andrea Conchado Peiró, Ana Isabel Sánchez Galdón, Rubén Ruiz García, María del Carmen Bas Cerdá, Elena Vázquez Barrachina, Rosa María Alcover Arándiga, Andrés Carrión García y José Miguel Carot Sierra

trabajos de cursos pasados, abundante documentación al respecto y los manuales del uso del software en este caso específico.

Ejemplos. Los alumnos pueden trabajar de forma individual o colaborativa aunque se intenta que sea esta última modalidad. Esta actividad tiene un peso relevante en la calificación final de la asignatura, pues supone la práctica totalidad de la nota final. Para su evaluación se recogen los informes escritos (memoria) y se califican utilizando la siguiente rúbrica:

Tabla 1. Rúbrica para la evaluación de la competencia Análisis y resolución de problemas

\begin{tabular}{|c|c|c|c|c|c|}
\hline & No asimilada & Poco asimilada & Asimilada & Bien asimilada & Muy bien asimilada \\
\hline $\begin{array}{l}\text { Originalidad } \\
\text { de la } \\
\text { propuesta }\end{array}$ & $\begin{array}{l}\text { El trabajo no contiene } \\
\text { ningún elemento original } \\
\text { ni plantea una estructura } \\
\text { diferente a la aportada } \\
\text { por el profesor o } \\
\text { compañeros }\end{array}$ & $\begin{array}{l}\text { El trabajo contiene } \\
\text { algunos elementos } \\
\text { originales, pero no tienen } \\
\text { relación ni aportan } \\
\text { información relevante al } \\
\text { problema planteado }\end{array}$ & $\begin{array}{l}\text { El trabajo contiene } \\
\text { algunos elementos } \\
\text { originales cuya } \\
\text { introduccion en el trabajo } \\
\text { aporta un nuevo enfoque } \\
\text { para la selección de la } \\
\text { mejor solución y la } \\
\text { interpretación de } \\
\text { resultados }\end{array}$ & $\begin{array}{l}\text { El trabajo está diseñado } \\
\text { con originalidad, con } \\
\text { elementos que aportan un } \\
\text { nuevo enfoque, y una } \\
\text { estructura novedosa para } \\
\text { la selección de la mejor } \\
\text { solución y la } \\
\text { interpretación de } \\
\text { resultados }\end{array}$ & $\begin{array}{l}\text { El trabajo destaca por la } \\
\text { originalidad del } \\
\text { planteamiento, los } \\
\text { elementos que incorpora } \\
\text { y la estructura para la } \\
\text { selección de la mejor } \\
\text { solución e interpretación } \\
\text { de resultados }\end{array}$ \\
\hline $\begin{array}{l}\text { Grado de } \\
\text { desarrollo } \\
\text { del trabajo }\end{array}$ & $\begin{array}{l}\text { El trabajo no es } \\
\text { coherente con los } \\
\text { conceptos básicos de la } \\
\text { asignatura ni aporta una } \\
\text { solución válida para el } \\
\text { problema planteado }\end{array}$ & $\begin{array}{l}\text { El trabajo muestra cierta } \\
\text { coherencia con los } \\
\text { conceptos básicos de la } \\
\text { asignatura, pero la } \\
\text { solución no es válida } \\
\text { para la resolución del } \\
\text { problema planteado. Se } \\
\text { observan graves lagunas } \\
\text { de comprensión. }\end{array}$ & $\begin{array}{l}\text { El trabajo muestra } \\
\text { coherencia con los } \\
\text { conceptos básicos de la } \\
\text { asignatura y el problema } \\
\text { planteado. Sin embargo, } \\
\text { la solución propuesta no } \\
\text { es la óptima para la } \\
\text { resolución del problema. } \\
\text { No se valoran soluciones } \\
\text { alternativas. }\end{array}$ & $\begin{array}{l}\text { El trabajo muestra } \\
\text { coherencia con los } \\
\text { conceptos básicos y } \\
\text { avanzados de la } \\
\text { asignatura, así como con } \\
\text { el problema planteado. } \\
\text { Se evalúan distintas } \\
\text { soluciones y se } \\
\text { selecciona una de ellas } \\
\text { argumentando las } \\
\text { razones }\end{array}$ & $\begin{array}{l}\text { El trabajo muestra } \\
\text { coherencia con los } \\
\text { conceptos básicos y } \\
\text { avanzados del área de } \\
\text { estudio, así como con el } \\
\text { problema planteado. Se } \\
\text { evalúan distintas } \\
\text { soluciones y se } \\
\text { selecciona correctamente } \\
\text { la solución óptima, } \\
\text { argumentando las } \\
\text { razones }\end{array}$ \\
\hline $\begin{array}{l}\text { Grado de } \\
\text { dificultad de } \\
\text { la propuesta }\end{array}$ & $\begin{array}{l}\text { La incoherencia de la } \\
\text { propuesta muestra que no } \\
\text { se han comprendido los } \\
\text { conceptos básicos }\end{array}$ & $\begin{array}{l}\text { La simplicidad de la } \\
\text { propuesta muestra una } \\
\text { comprensión muy } \\
\text { limitada de los conceptos } \\
\text { básicos }\end{array}$ & $\begin{array}{l}\text { La propuesta muestra } \\
\text { comprensión sustancial } \\
\text { de los conceptos básicos }\end{array}$ & $\begin{array}{l}\text { La complejidad de la } \\
\text { propuesta muestra una } \\
\text { comprensión completa } \\
\text { de los conceptos básicos } \\
\text { y avanzados }\end{array}$ & $\begin{array}{l}\text { La complejidad de la } \\
\text { propuesta muestra una } \\
\text { comprensión completa } \\
\text { de los conceptos básicos } \\
\text { y avanzados así como las } \\
\text { interrelaciones que se } \\
\text { establecen entre ellos }\end{array}$ \\
\hline $\begin{array}{l}\text { Calidad de } \\
\text { la memoria } \\
\text { presentada }\end{array}$ & $\begin{array}{l}\text { No se definen los } \\
\text { objetivos del trabajo ni } \\
\text { hay relación con los } \\
\text { contenidos de la } \\
\text { asignatura. No se } \\
\text { interpretan los resultados } \\
\text { obtenidos. Hay textos } \\
\text { confusos y faltas de } \\
\text { ortografía } \\
\text { sistemáticamente. El } \\
\text { formato no es adecuado } \\
\text { en absoluto. }\end{array}$ & $\begin{array}{l}\text { La definición de } \\
\text { objetivos del trabajo es } \\
\text { insuficiente y poco } \\
\text { relacionada con los } \\
\text { contenidos. La } \\
\text { interpretación de } \\
\text { reusltados no está } \\
\text { completa o tiene poca } \\
\text { calidad (poco } \\
\text { argumentada, falta de } \\
\text { razonamientos...) Hay } \\
\text { textos difíciles de } \\
\text { comprender y muchas } \\
\text { faltas de ortografía. Gran } \\
\text { parte del formato no es } \\
\text { adecuado }\end{array}$ & $\begin{array}{l}\text { Los objetivos se definen } \\
\text { de forma superficial y } \\
\text { con poca relación con los } \\
\text { contenidos. Se } \\
\text { interpretan todos los } \\
\text { resultados obtenidos, } \\
\text { pero mediante } \\
\text { comentarios simples y } \\
\text { poco desarrollados. }\end{array}$ & $\begin{array}{l}\text { Los objetivos se definen } \\
\text { con suficiente nivel de } \\
\text { detalle y se identifican } \\
\text { con claridad los } \\
\text { contenidos asociados a } \\
\text { cada objetivo. Se } \\
\text { interpretan todos los } \\
\text { resultados obtenidos } \\
\text { mediante comentarios } \\
\text { bien argumentados y } \\
\text { desarrollados. }\end{array}$ & $\begin{array}{l}\text { Los objetivos se definen } \\
\text { tras una profunda } \\
\text { reflexión sobre los } \\
\text { contenidos de la } \\
\text { asignatura, identificando } \\
\text { con claridad los } \\
\text { conceptos aplicables para } \\
\text { resolver el problema. Se } \\
\text { interpretan todos los } \\
\text { resultados obtenidos } \\
\text { mediante comentarios } \\
\text { que evidencian la } \\
\text { reflexión y se valoran } \\
\text { distintas soluciones } \\
\text { presentando puntos } \\
\text { fuertes y débiles. }\end{array}$ \\
\hline
\end{tabular}

(cc)) EY-NC-ND 2015, Universitat Politècnica de València

Congreso In-Red (2015) 
Propuesta de actividades de enseñanza - aprendizaje e instrumentos de evaluación de competencias en el Máster Universitario en Ingeniería de Análisis de Datos, Mejora de Procesos y Toma de

Decisiones

\begin{tabular}{|c|c|c|c|c|c|}
\hline $\begin{array}{l}\text { Calidad de } \\
\text { la } \\
\text { presentación } \\
\text { oral del } \\
\text { trabajo }\end{array}$ & $\begin{array}{l}\text { No participa en la } \\
\text { presentación oral }\end{array}$ & $\begin{array}{l}\text { No expresa con claridad } \\
\text { los objetivos del trabajo } \\
\text { ni los resultados } \\
\text { obtenidos. La } \\
\text { presentación no está bien } \\
\text { organizada y es díficil de } \\
\text { seguir. No se utiliza el } \\
\text { lenguaje correcto desde } \\
\text { el punto de vista } \\
\text { gramatical, ni del léxico } \\
\text { de la asignatura. Es } \\
\text { evidente que no ha } \\
\text { preparado la } \\
\text { presentación. }\end{array}$ & $\begin{array}{l}\text { Se enuncian y exponen } \\
\text { los objetivos y resultados } \\
\text { del trabajo con claridad } \\
\text { pero poco nivel de } \\
\text { detalle. La presentación } \\
\text { está bien organizada. El } \\
\text { lenguaje utilizado es } \\
\text { correcto gramaticalmente } \\
\text { pero no se utiliza el } \\
\text { léxico de la asignatura. } \\
\text { No se permite apreciar la } \\
\text { complejidad del trabajo a } \\
\text { través de la presentación. }\end{array}$ & $\begin{array}{l}\text { Se enuncian y exponen } \\
\text { los objetivos y resultados } \\
\text { de la asignatura con } \\
\text { suficiente nivel de } \\
\text { detalle. Asimismo se } \\
\text { aportan comentarios y } \\
\text { argumentaciones sobre } \\
\text { los resultados obtenidos. } \\
\text { La presentación está bien } \\
\text { organizada, es coherente } \\
\text { y puede seguirse con } \\
\text { facilidad. El lenguaje } \\
\text { utilizado es correcto } \\
\text { gramaticalmente. Utiliza } \\
\text { correctamente el léxico } \\
\text { de la asignatura. }\end{array}$ & $\begin{array}{l}\text { Se enuncian } \\
\text { detalladamente los } \\
\text { objetivos y resultados de } \\
\text { la asignatura, } \\
\text { relacionándolos con } \\
\text { conceptos de otras áreas } \\
\text { o contextos. Se aportan } \\
\text { comentarios y } \\
\text { argumentaciones que } \\
\text { justifican los resultados } \\
\text { obtenidos, } \\
\text { fundamentados en } \\
\text { investigaciones y/o } \\
\text { experiencias previas. La } \\
\text { presentación está bien } \\
\text { oganizada, es coherente } \\
\text { y fácil de seguir. } \\
\text { También demuestra } \\
\text { originalidad y } \\
\text { creatividad. El lenguaje } \\
\text { utilizado es correcto } \\
\text { gramaticalmente. Se } \\
\text { utiliza el léxico de la } \\
\text { asignatura y se } \\
\text { introducen nuevos } \\
\text { términos relacionados } \\
\text { con conceptos avanzados } \\
\text { del área }\end{array}$ \\
\hline $\begin{array}{l}\text { Capacidad a } \\
\text { la hora de } \\
\text { contestar las } \\
\text { preguntas } \\
\text { de los } \\
\text { compañeros } \\
\text { y del } \\
\text { profesor }\end{array}$ & $\begin{array}{l}\text { Se observa un } \\
\text { desconocimiento total } \\
\text { sobre el contenido del } \\
\text { trabajo }\end{array}$ & $\begin{array}{l}\text { Se observa un bajo nivel } \\
\text { de comprensión del } \\
\text { contenido del trabajo }\end{array}$ & $\begin{array}{l}\text { Se observa un nivel de } \\
\text { comprensión aceptable } \\
\text { del contenido del trabajo, } \\
\text { aunque algunas } \\
\text { respuestas han sido } \\
\text { dubitativas }\end{array}$ & $\begin{array}{l}\text { Se observa un nivel de } \\
\text { comprensión aceptable } \\
\text { del contenido del trabajo, } \\
\text { respondiendo con } \\
\text { seguridad a todas las } \\
\text { preguntas }\end{array}$ & $\begin{array}{l}\text { Se observa un } \\
\text { conocimiento total del } \\
\text { contenido del trabajo y } \\
\text { que domina los } \\
\text { conceptos que se } \\
\text { plantean en las preguntas }\end{array}$ \\
\hline
\end{tabular}

Las calificaciones obtenidas por los alumnos evidenciaron mayor un dominio de la competencia tras la resolución del segundo trabajo. Los 20 alumnos matriculados en la asignatura obtuvieron una nota promedio significativamente inferior (8.22) en el primer problema, frente a la obtenida en el segundo problema (8.87), mediante la prueba t de Student para muestras relacionadas $(\mathrm{t}=-4.46, \mathrm{p}=0.000)$. La correlación entre ambas calificaciones alcanzó el valor de 0.877 , indicando una fuerte relación entre las notas obtenidas en ambos problemas. Asimismo, el diagrama de dispersión mostrado en la Figura 1 representa esta fuerte relación lineal. Aún siendo una muestra pequeña, se observa que durante el transcurso de la materia, ha habido una mejora en las notas obtenidas en el segundo trabajo. Se observa también que, al ser un Máster donde los estudiantes tienen una formación de base muy heterogénea, hay una cierta dispersión en las notas. La alta correlación implica el efecto fuerte de esta formación de base sobre la capacidad del estudiante a la hora de resolver ambos trabajos. 
Andrea Conchado Peiró, Ana Isabel Sánchez Galdón, Rubén Ruiz García, María del Carmen Bas Cerdá, Elena Vázquez Barrachina, Rosa María Alcover Arándiga, Andrés Carrión García y José Miguel Carot Sierra

Figura 1. Diagrama de dispersión de las calificaciones obtenidas por los alumnos mediante la aplicación de la rúbrica

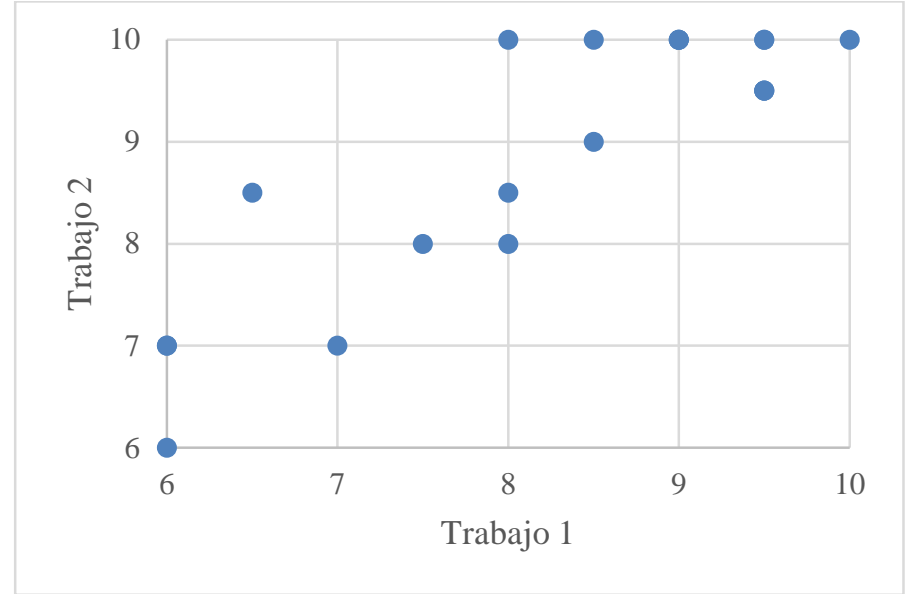

\section{Conclusiones}

El presente trabajo ha permitido replantear internamente las competencias básicas y generales del título, logrando una mayor adaptación a las competencias transversales propuestas por la UPV a nivel institucional. Por otro lado, se ha propuesto una reagrupación de las competencias específicas, acorde con el nivel de concreción exigido para competencias y resultados del aprendizaje. A partir de esta reformulación de las competencias que pueden adquirirse en el título, se ha definido una actividad de enseñanza - aprendizaje y se ha creado una rúbrica para su evaluación. El objetivo de ambos elementos es contribuir y medir la adquisición de la competencia transversal Análisis y resolución de problemas y la competencia específica Elaboración, construcción, validación e interpretación de modelos. La aplicación de este instrumento para la evaluación de los trabajos de los alumnos, ha permitido identificar diferencias significativas entre la resolución del primer y el segundo problema propuesto por el profesor. Como futuras líneas de investigación, se propone realizar un análisis de ítems para evaluar la fiabilidad y validez de la rúbrica, como instrumento de evaluación de las competencias seleccionadas.

(c)) EY-NC-ND 2015, Universitat Politècnica de València

Congreso In-Red (2015) 
Propuesta de actividades de enseñanza - aprendizaje e instrumentos de evaluación de competencias en el Máster Universitario en Ingeniería de Análisis de Datos, Mejora de Procesos y Toma de

Decisiones

\section{Agradecimientos}

El desarrollo de esta innovación ha sido posible gracias al Proyecto de Innovación y Mejora Educativa «Identificación y análisis de dimensiones competenciales para el diseño de actividades e instrumentos de evaluación orientados a competencias» (PIME/2014/A/010/B), evaluado por la Agencia Valenciana d’Avaluació i Prospectiva (AVAP) y concedido por la Comisión de Evaluación y Seguimiento de Proyectos de Innovación y Convergencia (CESPIC) de la Universitat Politècnica de València.

\section{Referencias}

Agencia Nacional de Evaluación de la Calidad y Acreditación [ANECA] (2013). Guía para la redacción, puesta en práctica y evaluación de los resultados del aprendizaje. Madrid: ANECA.

Biggs, J. (2006). Calidad del aprendizaje universitario. Madrid: Narcea.

De Miguel, M. (Coord.) (2006). Metodologías de enseñanza y aprendizaje para el desarrollo de competencias. Orientaciones para el profesorado universitario ante el espacio europeo de educación superior. Madrid: Alianza Editorial.

Fernández, A. (2010). La evaluación orientada al aprendizaje en un modelo de formación por competencias en la educación universitaria. Revista de Docencia Universitaria, 8(1): 11-34.

Universidad Politécnica de Valencia (2014). Proyecto Institucional de Incorporación de las competencias transversales en los curricula de los egresados UPV [documento interno].

Villardón - Gallego, L. (2015). Competencias genéricas en Educación Superior. Madrid: Narcea. 